OPEN ACCESS

Edited by:

Brian Peer,

Western Illinois University,

United States

Reviewed by:

Virginia Abernathy,

Clemson University, United States

Eleanor Caves,

University of California,

Santa Barbara, United States

Tanmay Dixit,

University of Cambridge,

United Kingdom, in collaboration with

reviewer EC

*Correspondence:

Daniel Hanley

dhanley2@gmu.edu

Specialty section:

This article was submitted to Coevolution,

a section of the journal

Frontiers in Ecology and Evolution

Received: 30 April 2021

Accepted: 14 October 2021

Published: 04 November 2021

Citation:

Hanley D, Rutledge SL and Villa $J$ (2021) The Perceptual and Cognitive Processes That Govern Egg Rejection

in Hosts of Avian Brood Parasites.

Front. Ecol. Evol. 9:702934.

doi: 10.3389/fevo.2021.702934

\section{The Perceptual and Cognitive Processes That Govern Egg Rejection in Hosts of Avian Brood Parasites}

\author{
Daniel Hanley ${ }^{1 *}$, Samantha L. Rutledge $\mathrm{e}^{2,3}$ and Juliana Villa ${ }^{1}$ \\ ${ }^{1}$ Department of Biology, George Mason University, Fairfax, VA, United States, ${ }^{2}$ Department of Biology, Mansfield University \\ of Pennsylvania, Mansfield, PA, United States, ${ }^{3}$ Museum of Natural Science, Louisiana State University, Baton Rouge, LA, \\ United States
}

Hosts of avian brood parasites are under intense selective pressure to prevent or reduce the cost of parasitism. Many have evolved refined egg discrimination abilities, which can select for eggshell mimicry in their parasite. A classic assumption underlying these coevolutionary dynamics is that host egg recognition depends on the perceivable difference between their own eggs and those of their parasite. Over the past two decades, the receptor noise-limited (RNL) model has contributed to our understanding of these coevolutionary interactions by providing researchers a method to predict a host's ability to discriminate a parasite's egg from its own. Recent research has shown that some hosts are more likely to reject brown eggs than blue eggs, regardless of the perceived differences to their own. Such responses suggest that host egg recognition may be due to perceptual or cognitive processes not currently predictable by the RNL model. In this perspective, we discuss the potential value of using the RNL model as a null model to explore alternative perceptual processes and higher-order cognitive processes that could explain how and why some hosts make seemingly counter-intuitive decisions. Further, we outline experiments that should be fruitful for determining the perceptual and cognitive processing used by hosts for egg recognition tasks.

Keywords: brood parasitism, color categorization, egg rejection, opponent channels, receptor-noise limited model

\section{INTRODUCTION}

Avian brood parasitism is an alternative reproductive strategy where one female lays her eggs in another bird's nest, imposing the costs of rearing her young on a set of foster parents (Stevens, 2013). In response, hosts often evolve egg recognition abilities as a major line of defense against parasitism, which, in turn, can select for improved eggshell mimicry in their parasite and instigate a coevolutionary arms race (Dawkins and Krebs, 1979; Davies and Brooke, 1989; Stoddard and Stevens, 2011). Such interactions have provided researchers with a tractable system to examine parasitic relationships and coevolution (Dawkins and Krebs, 1979; Davies and Brooke, 1989; Hauber et al., 2015b).

The perceptual and cognitive processes governing host egg recognition are central to understanding host decision-making and coevolutionary arms races (Dawkins and Krebs, 1979; 
Davies and Brooke, 1989; Hauber et al., 2015b). Classic theory assumes that hosts can select for eggshell mimicry in the parasite by rejecting parasite eggs they perceive as dissimilar to their own (Dawkins and Krebs, 1979). Pioneering work by Stoddard and Stevens (2011) applied an avian receptor noise limited (RNL) model (Vorobyev and Osorio, 1998) to the common cuckoo, Cuculus canorus, and their respective hosts. They found that hosts with strong recognition abilities were parasitized by cuckoos that laid eggs with refined eggshell mimicry (as predicted by the RNL model). However, while useful for predicting discriminability between stimuli (Olsson et al., 2018; Price et al., 2019), the RNL model's original intent was to make predictions with which to compare behavioral responses and then infer color processing mechanisms (Osorio and Vorobyev, 2018). Contrary to traditional expectations (Brooke and Davies, 1988; Reeve, 1989), Hanley et al. (2017) illustrated that some hosts reject brown model eggs more than blue model eggs, even when those eggs were equally dissimilar to the hosts' own. In this experiment, model eggs were painted colors that specifically aligned with perceived variation in natural eggshell color (from blue-green to brown; Hanley et al., 2015). This finding has been meta-replicated in five hosts from three continents, each of which face distinct forms of parasitism (Abolins-Abols et al., 2019; Hanley et al., 2019b; Manna et al., 2020); however, not all hosts will accept any egg models painted "blue" (Langmore et al., 2005; Begum et al., 2012; Hanley et al., 2019a; Abernathy et al., 2021). These counterintuitive and, at times disparate, findings raise the question, "why have these hosts deviated from our null expectations"?

Here, we explore alternative visual-cognitive processes that may explain such deviations from the expected RNL model. Specifically, we describe color vision in birds by explaining how color is both received and perceived. We then outline the discrimination challenge facing hosts and describe a higher-level cognitive process (categorization) that hosts may use to process this color information during decision-making (Harnad, 1987; Shepard, 1987; Goldstone and Hendrickson, 2009). Our goal is to contextualize previous findings of strikingly similar colorbiased rejection behaviors by describing egg recognition tasks from color reception through perception (Figure 1). Finally, we provide potential experimental frameworks to aid future investigations into the perceptual and cognitive processes used by hosts for egg discrimination tasks.

\section{Color Reception}

Birds are thought to have excellent color vision (Jacobs, 1981; Goldsmith, 1990; Hart, 2001; Ödeen and Håstad, 2003). They possess four types of single-cone photoreceptors, sensitive to ultraviolet (or violet), short, medium, or long wavelength light (Hart, 2001). Avian color reception is thought to be based on the stimulation of these single cones (Hart, 2001; Ödeen and Håstad, 2003), where estimates of quantum catches (i.e., the number of photons being absorbed by the cone from the stimulus) approximate the stimulation of each photoreceptor:

$$
Q_{i}=\int_{300}^{700} R_{i}(\lambda) S(\lambda) I(\lambda) O(\lambda) d \lambda
$$

Where $Q_{i}$ represents the quantum catch for receptor $i, R$ is the sensitivity of photoreceptor $i$ after accounting for oil droplet transmittance, $S$ is the reflectance of a stimulus, $I$ is the irradiance in photon flux, and $O$ is the ocular media that narrows the sensitivity of photoreceptors in birds (Bowmaker et al., 1997; Vorobyev, 2003). By convention, we refer to the quantum catches of the ultraviolet/violet-, short-, medium-, and long-wavelength sensitive photoreceptors as $Q_{u}, Q_{s}, Q_{m}$, and $Q_{l}$, respectively.

\section{Color Perception}

Before a color can give rise to a perceptual experience in an organism, the color that is received by the eye must be transformed and transmitted to the brain (Osorio et al., 1999; Kelber, 2016; Price et al., 2019). This is done by forming opponent channels that contrast received quantum catches against each other (Figure 1), a process that underlies color discrimination in a range of organisms (Osorio et al., 1999; Ventura et al., 2001; Rocha et al., 2008; Kelber, 2016; Price et al., 2019). In theory, related opponent channels [e.g., $\left(Q_{u}+Q_{l}\right)-\left(Q_{s}+Q_{m}\right)$ and $\left.\left(Q_{s}+Q_{m}\right)-\left(Q_{u}+Q_{l}\right)\right]$ can produce signals that vary in sign (positive or negative) and therefore may result in two different responses. Unfortunately, the actual neural mechanisms of each channel-and, indeed, the total number of channels used-are unknown in birds (Kelber, 2016; Price and Fialko, 2018; Vasas et al., 2018; Price et al., 2019). This makes it challenging to model the actual signals reaching the avian brain.

To circumvent this difficulty, Vorobyev and Osorio (1998) developed the RNL model that predicts discriminability between stimuli. In this model, the actual opponent channels are unimportant and the sign of the signal makes no difference on model output (Vorobyev and Osorio, 1998). This model assumes that visual signals and discrimination thresholds are determined by photoreceptor noise (Vorobyev and Osorio, 1998), that there are one fewer opponent channels than photoreceptor types, and that altering light levels do not change perceived differences between stimuli (Kelber, 2016). The RNL model predicts differences between stimuli in just noticeable differences (JNDs), classifying stimuli as either discriminable (>1 JND) or not discriminable $(<1 \mathrm{JND})$. Although in practice, it is generally recognized that the theoretical threshold of 1 JND is likely overly conservative in most natural contexts, and more realistic thresholds may be set $\sim 2$ JNDs (Spottiswoode and Stevens, 2010, 2012; Stevens et al., 2013). This model is appealing because it accurately estimates visible contrast, at least in some contexts (e.g., brightly lit conditions), provided reflectance spectra for two stimuli (e.g., those in a detection or discrimination task), spectral sensitivity estimates of each of the organism's photoreceptor types (Price et al., 2019), and the irradiance of the environment (Endler, 1993) are available.

However, there are certain conditions that may impact the RNL model's ability to accurately predict discriminability. For example, this model does not currently account for how visual contrast against the background impacts the thresholds beyond which two stimuli can be discriminated (i.e., for discrimination tasks). When viewing colorful stimuli, most animals will compensate for differences in light conditions through a process known as chromatic adaptation (Lind, 2016; Price et al., 2019). 


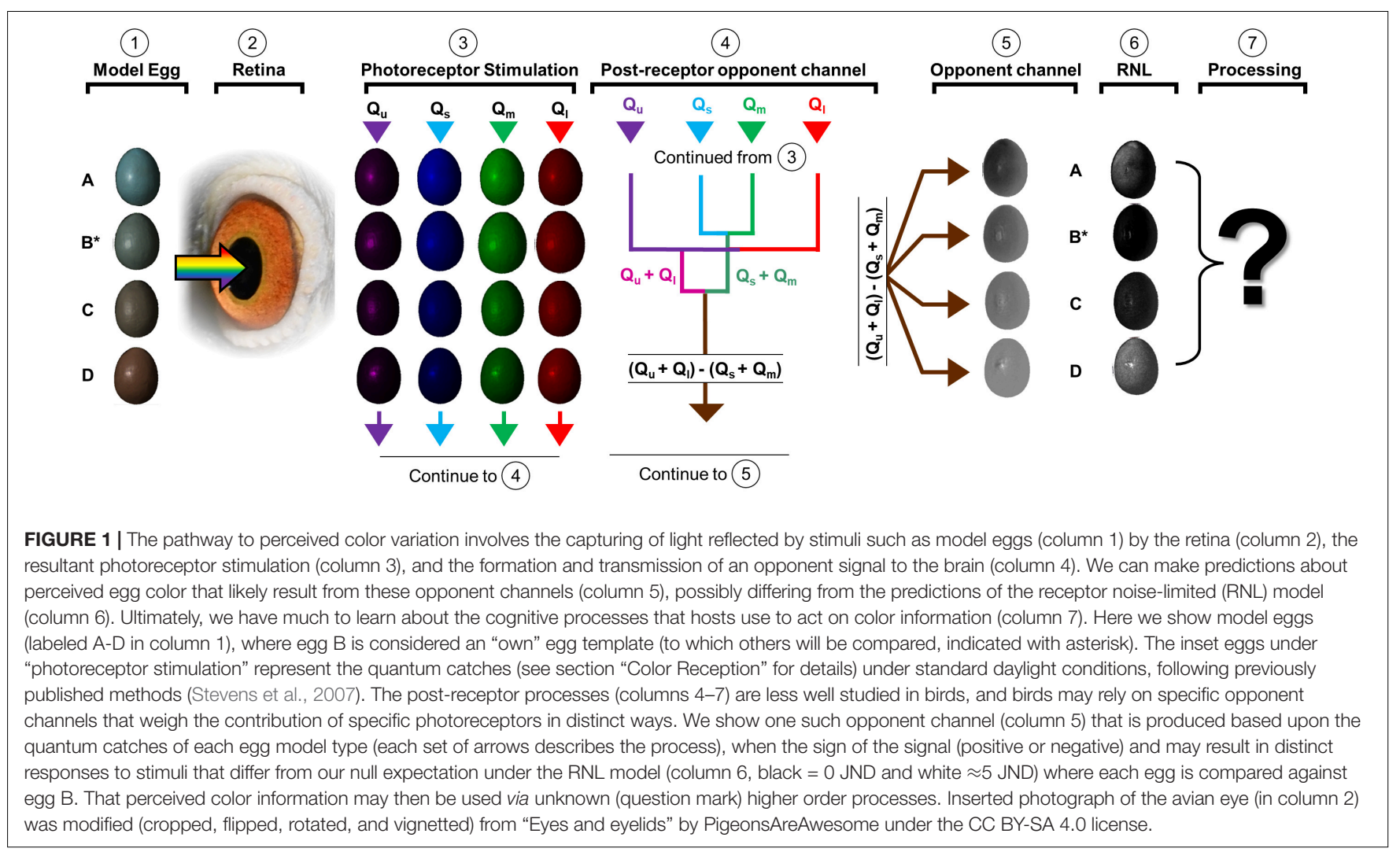

In this process, when the background predominantly reflects long-wavelength light (typical in birds' nests), the viewer will upregulate short-wavelength receptor signals (e.g., blue) and downregulate long-wavelength receptor signals (e.g., brown; Price et al., 2019). Consequently the predicted detection of bluegreen eggs in brown nests is, rightly, improved (higher JND) by accounting for the nest background (Price et al., 2019). However, animals tend to perform better on discrimination tasks (e.g., comparing a parasite's egg and a host's egg) when stimuli are more similar to the background (e.g., two brown eggs on a brown nest) and poorer when stimuli are more different from the background (e.g., two blue eggs on a brown nest); thus, chromatic adaptation can effectively alter the threshold necessary to differentiate stimuli (Krauskopf and Gegenfurtner, 1992; Lind, 2016; Price et al., 2019). Currently, the RNL model does not account for these effects for discrimination tasks.

\section{Higher-Order Processing}

Most studies on color discrimination assume that animals use low-level cognitive mechanisms simply based on discrimination thresholds (Kelber and Osorio, 2010); however, previous research has provided evidence of decision-rules based on higher-level cognitive mechanisms in invertebrates, fish, mammals, and even in birds (Tapper and Halpern, 1968; Sandell et al., 1979; Nelson and Marler, 1989; Poralla and Neumeyer, 2006; Ham and Osorio, 2007; Benard and Giurfa, 2008; Avarguès-Weber et al., 2011; Lachlan and Nowicki, 2015; Renoult et al., 2015; Caves et al., 2018). One such higher-level cognitive process is categorization, which occurs when responses to stimuli vary less within categories than between categories (Repp, 1984; Harnad, 1987; Treisman et al., 1995). This is known as a boundary effect, which is a hallmark test of categorical perception and can only be assessed measuring responses across a phenotypic range that spans both sides of a decision boundary (Harnad, 1987; ten Cate and Rowe, 2007; Kelber and Osorio, 2010; Hauber et al., 2015b). Categorization is thought to increase the speed, accuracy, and certainty of choices, while reducing the requirements for neural processing (Nelson and Marler, 1989; Kepecs et al., 2008), particularly useful for performing unfamiliar tasks or when information is uncertain (Dukas and Waser, 1994; Benard et al., 2006; Kepecs et al., 2008). Although the potential for hosts to use color categorization in egg recognition has been discussed previously (Spottiswoode and Stevens, 2010; Hanley et al., 2017), and tests have illustrated a clear decision boundary (see figures S3 and S4 from, Hanley et al., 2017), no study has yet confirmed that hosts use categorization for egg recognition (Green et al., 2020).

\section{DISCUSSION}

By providing reasonable predictions about perceivable differences between host and parasite eggs, the RNL model has proven a crucial method for testing certain hypotheses surrounding coevolutionary arms races between host perception and parasite egg phenotypes (Spottiswoode and Stevens, 2010; Stoddard and Stevens, 2011). Comparisons between the predictions of the RNL model and actual host responses can demonstrate 
higher-order processes, such as categorization (Nelson and Marler, 1989; Caves et al., 2018), or help determine the discrimination thresholds necessary for specific egg recognition tasks (Lind, 2016; Olsson et al., 2020). Such experiments would refine our understanding of the perceptual and cognitive processes that underly egg recognition decisions (e.g., opponent channels, chromatic adaptation, and categorization) and advance our understanding of host-parasite coevolutionary dynamics. Here we outline tests necessary to explore hitherto untested perceptual and cognitive processes, which may explain why some hosts' behaviors appear to deviate from the expectations of the RNL model (e.g., rejecting brown but accepting equally dissimilar blue eggs).

\section{Host Selection for Proposed Tests}

The experiments we outline below are ideally suited for hosts traditionally considered intermediate rejecters (Rothstein, 1975; Davies and Brooke, 1989; Peer and Sealy, 2004), rather than hosts that unilaterally accept or reject eggs. Additionally, these tests are suited for hosts with intermediate, rather than extreme, eggshell colors so that experimental egg model stimuli can be realistically bluer or browner than the host's. In such an experiment, a researcher would insert a single egg model, either experimental or control, into each host's nest and record their response (reject/accept) within a predefined period (Canniff et al., 2018). We advocate that researchers estimate avian-perceived coloration of each host egg (rather than average host color as in Hanley et al., 2017), experimental egg, and nest, in addition to measuring the irradiance at each nest, as these data are vital for interpreting host behavioral responses.

\section{Perceptual Mechanisms}

Egg rejection studies using egg models varying in coloration along a continuous range would be particularly useful for determining whether a host's response is governed by a particular opponent channel (Hanley et al., 2017, 2019b; Abolins-Abols et al., 2019; Manna et al., 2020). Such experiments (Figure 2A) would allow a researcher to determine whether host response is better predicted by opponent channel(s) or discriminable differences predicted under the RNL model and also whether responses vary sharply anywhere along that color range (decision boundary, see below). This could be a fruitful line of research, since past studies have found that individual photoreceptor quantum catches provided better predictions of host response than discriminable differences predicted under the RNL model (Cassey et al., 2008; Hauber et al., 2020). If hosts used a common opponent channel, that may explain why disproportionate rejections of brown eggs have been found in a range of hosts (Stokke et al., 2007; Cassey et al., 2008; Soler et al., 2012; Hanley et al., 2017, 2019b; Abolins-Abols et al., 2019; Manna et al., 2020). If such a common channel exists, and is used for egg recognition, it most likely broadly distinguishes shortfrom long-wavelength dominated colors (e.g., blue and brown, respectively). Still, while uncovering a specific shared opponent channel used by diverse hosts would be exciting, other aspects of visual perception might also explain host behaviors that deviate from our null expectations.
Chromatic adaptation to the (typically) brown nest background provides one such intriguing possibility. Lind (2016) demonstrated that the thresholds necessary for color discrimination can shift depending on the contrast between the color of the stimuli and the nest background. Thus, hosts with blue-green eggs would face a comparatively more challenging task when discriminating a blue egg (i.e., higher discrimination threshold) than discriminating a brown egg (i.e., lower discrimination threshold). Consistent with this observation, hosts found to disproportionately reject brown egg models laid (at least moderately) blue-green eggs (Honza et al., 2007; Cassey et al., 2008; Soler et al., 2012; Hanley et al., 2017, 2019b; Abolins-Abols et al., 2019; Manna et al., 2020). Although past experiments found that nest contrast did not influence host egg recognition (Aidala et al., 2015; Hauber et al., 2015a), a blue nest lining did increase performance on an egg discrimination task by $25 \%$ in the blue-green egg laying American robin Turdus migratorius (Aidala et al., 2015). We feel this is still a promising line of research, particularly if future studies employ an experimental manipulation of nest background (Aidala et al., 2015) alongside sufficient variation in the color of egg stimuli to determine threshold values (sensu Lind, 2016). We suggest measuring the discrimination thresholds by recording behavioral responses to sets of blue-green and brown egg stimuli sufficiently diverse to surpass threshold levels (e.g., 66.7\% rejection rates; see Figure 2B) on high and low contrast nest backgrounds.

\section{Cognitive Mechanisms}

If host responses show evidence of a sharp decision boundary (Figure 2A), subsequent experiments should test for color categorization. We suggest deploying discrete sets of egg models of carefully designed colors (Nelson and Marler, 1989; Cheke et al., 2006; Ham and Osorio, 2007; Caves et al., 2018), rather than eggs with continuously varying colors (sensu Hanley et al., 2017). For example, egg sets could be bluer (set 1) and browner (set 2) than the hosts' own (Figure 2C), but of identical brightness. Within each set, one stimulus (stimulus 1) should be relatively similar (e.g., 3 JND) to the host's own egg (the control stimulus) and differ from the next stimulus (stimulus 2) in the set by an identical degree (e.g., 3 JND). Importantly, sets of stimuli should span the previously detected decision boundary, and there must be comparable perceivable differences within and between categories. While our focus is on colors ranging from blue-green to brown, other color ranges can be explored and more colors can be added as necessary. Color categorization would be detected if differences in host responses are significantly greater across the category boundary (see above) than within either category. Although this is a necessary follow-up test to confirm color categorization in hosts (Green et al., 2020), it may not reveal the basis for such categorical behavior. Typically, categorical behavioral responses in an experiment such as the one we propose (Figure 2) would imply hosts assign categorical labels to distinguishable egg stimuli (e.g., egg sets); however, identical responses may result from other processes (e.g., chromatic adaptation altering discrimination thresholds for certain discriminations tasks; 

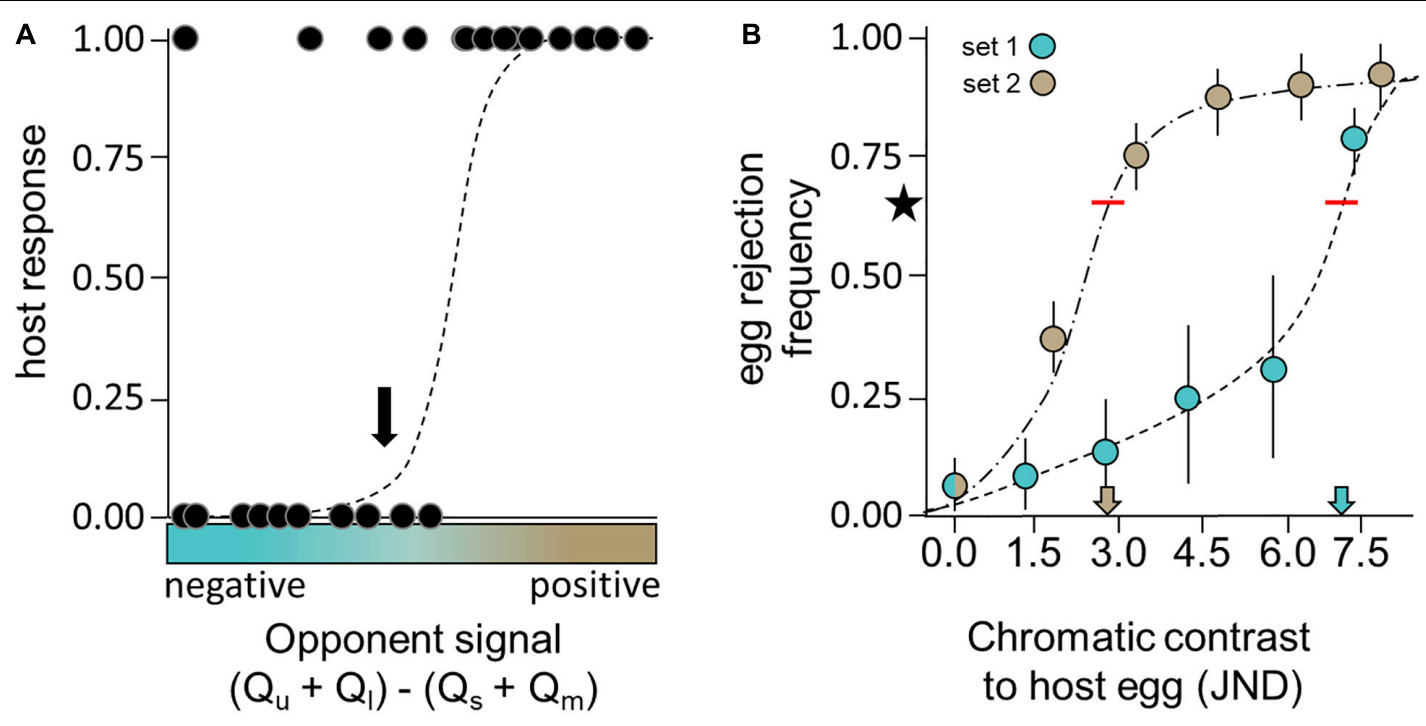

C

\section{Experimental design to detect categorization}
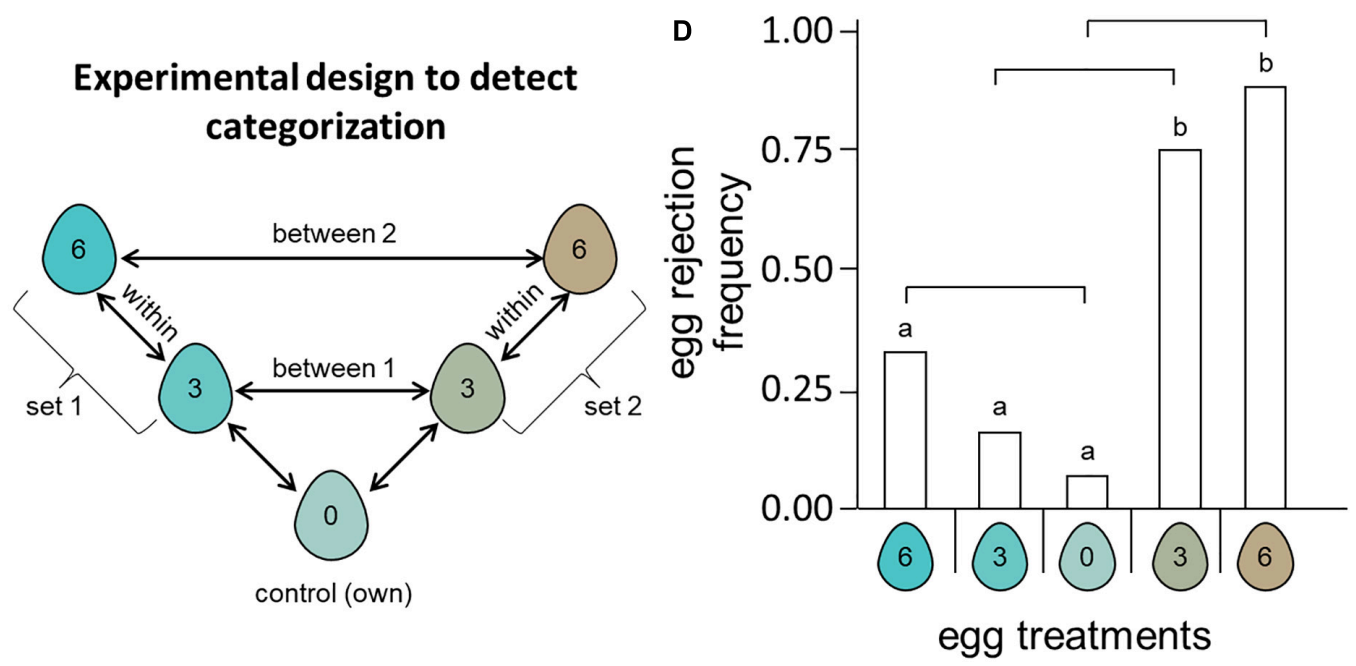

FIGURE 2 | Here we illustrate expected results from an experiment $\mathbf{( A )}$ investigating host response to eggs (acceptance $=0$, rejection $=1)$ painted colors ranging from bluer (negative) to browner (positive) than the hosts' own eggs (solid line on $\mathrm{x}$ axis). Egg stimuli are plotted by their opponent channel signal,

$\left(Q_{u}+Q_{l}\right)-\left(Q_{s}+Q_{m}\right)$, such that negative and positive values represent blue and brown egg colors, respectively. In this example, hosts reject eggs bluer than their own even less frequently than eggs with better color matches (as seen in Turdus thrushes, Hanley et al., 2017). We fit a hypothetical logistic regression (dashed line) to these data. These predictions would be compared against the predictions of an RNL model. For reference, an arrow indicates the color that most closely matches the host's own egg color. Such egg models (B) that vary from the host's own egg color (chromatic contrasts in JND) can be deployed in nests to measure the discrimination thresholds for eggs bluer (set 1) or browner (set 2) than the host's own. For a host with a moderately blue-green egg, we expect that chromatic adaptation to a natural brown nest background could shift the discrimination threshold (blue and brown arrows on x-axis), such that threshold necessary to detect $66.7 \%$ of egg models (red lines) vary for each set of stimuli. Here error bars represent variation across multiple experiments (e.g., spanning years or populations). Finally, we illustrate an (C) experimental schematic and set of (D) expected results for tests of color categorization. Eggs from each egg set, would vary by consistent intervals. The numbers in egg icons represent the chromatic contrast to the host's own egg, with the control model identical in color to the host's own. To detect categorization, the (D) differences between categories (egg sets) would have to be larger than differences within. We illustrate hypothetical results [identical to panel (B)] and the post hoc significance of these comparisons (above bars) that would document categorical behavior, emphasizing particularly informative comparisons using lines above the bars. These illustrate hypothetical results based on past results from hosts that lay blue-green eggs (e.g., Turdus thrushes: Hanley et al., 2017); however, these designs would be particularly informative on a host population that has a distinct egg polymorphism (relatively blue or relatively brown) as in the Daurian Redstart Phoenicurus auroreus (Yang et al., 2016; Zhang et al., 2021a,b).

Figures 2B,C). Regardless of the underlying mechanism of categorical behaviors (e.g., Caves et al., 2020), such rejection behavior would select for eggshell phenotypes in novel and unappreciated ways.

\section{Expected Evolutionary Outcomes}

Importantly, these perceptual and cognitive processes (i.e., chromatic adaptation, categorization) may all impact host egg recognition, and may not be mutually exclusive. Each can be 
tested by comparing host behavior to the predictions of the RNL model, and would impact host-parasite dynamics in interesting ways. For example, we would expect that heavily parasitized host populations that lay blue-green eggs would evolve even bluer eggs than unparasitized populations, under either categorization or chromatic adaptation, due to their preferential rejection of brown eggs (Soler et al., 2012; Hanley et al., 2013). In these cases, host discrimination might not only select for parasite eggshell mimicry, but also for shifts of the discrimination threshold itself (Figure 2B). We argue that these aspects of perception and cognition are as vital to coevolutionary arms races as the egg traits that are selected. Furthermore, we expect that the cognitive mechanisms underlying host egg recognition will be more plastic (Buchanan et al., 2008) than their egg and visual morphologies, which are, respectively, constrained and conserved traits (Ödeen and Håstad, 2003; Hanley et al., 2015). Focusing research attention on these more labile cognitive processes will likely be fruitful for determining the role hosts play in shaping coevolutionary dynamics.

\section{Conclusion}

In this perspective, we propose that hosts' discontinuous responses to continuous variation in eggshell color could be explained by more explicit perceptual (opponent channels, chromatic adaptation) and cognitive (categorization) models. By outlining fruitful directions for future research, we encourage researchers to use the RNL model as a null model with which to compare other higher-level processes. Avian brood parasitism provides an ideal system to not only learn about parasitism and coevolution (Soler and Soler, 2000; Stoddard and Hauber, 2017), but also the hitherto unidentified perceptual and cognitive

\section{REFERENCES}

Abernathy, V. E., Johnson, L. E., and Langmore, N. E. (2021). An experimental test of defenses against avian brood parasitism in a recent host. Front. Ecol. Evol. 9:1-14.

Abolins-Abols, M., Hanley, D., Moskát, C., Grim, T., and Hauber, M. E. (2019). Anti-parasitic egg rejection by great reed warblers (Acrocephalus arundinaceus) tracks differences along an eggshell color gradient. Behav. Proc. 166:103902. doi: 10.1016/j.beproc.2019.103902

Aidala, Z., Croston, R., Schwartz, J., Tong, L., and Hauber, M. E. (2015). The role of egg-nest contrast in the rejection of brood parasitic eggs. J. Exp. Biol. 218, 1126-1136. doi: $10.1242 /$ jeb. 108449

Avarguès-Weber, A., Deisig, N., and Giurfa, M. (2011). Visual cognition in social insects. Ann. Rev. Entomol. 56, 423-443. doi: 10.1146/annurev-ento-120709144855

Begum, S., Moksnes, A., Røskaft, E., and Stokke, B. G. (2012). Responses of potential hosts of Asian cuckoos to experimental parasitism. Ibis 154, 363-371. doi: 10.1111/j.1474-919x.2012.01213.x

Benard, J., and Giurfa, M. (2008). The cognitive implications of asymmetric color generalization in honeybees. Animal Cogn. 11, 283-293. doi: 10.1007/s10071007-0112-5

Benard, J., Stach, S., and Giurfa, M. (2006). Categorization of visual stimuli in the honeybee Apis mellifera. Animal Cogn. 9, 257-270. doi: 10.1007/s10071-0060032-9

Blackwell, B. F., Devault, T. L., Seamans, T. W., Lima, S. L., Baumhardt, P., and Fernández-Juricic, E. (2012). Exploiting avian vision with aircraft lighting to reduce bird strikes. J. Appl. Ecol. 49, 758-766. doi: 10.1111/j.1365-2664.2012. 02165.x mechanisms (Stoddard and Hauber, 2017) used by wild birds. By developing experiments explicitly designed to discern the underpinnings of avian vision, we can learn more about how birds interpret the world around them, an understanding that is vital to grasping their past, present, and future evolutionary trajectories (Endler and Mielke, 2005; Endler et al., 2005; Stevens, 2011), as well as to informing current conservation efforts (Blackwell et al., 2012; Dominoni et al., 2020). It is our hope that this manuscript will catalyze future investigations into the cognitive processes that underly perception and decision-making in broad taxonomic groups and contexts.

\section{DATA AVAILABILITY STATEMENT}

The original contributions presented in the perspective contain no data, further inquiries can be directed to the corresponding author/s.

\section{AUTHOR CONTRIBUTIONS}

$\mathrm{DH}$ provided the concept. DH, SR, and JV proposed experimental designs. All authors worked to develop and revise the writing.

\section{ACKNOWLEDGMENTS}

We would like to thank Brian Peer for the invitation to this special issue of Frontiers in Ecology and Evolution and helpful feedback from Alex Di Giovanni and three reviewers.

Bowmaker, J. K., Heath, L. A., Wilkie, S. E., and Hunt, D. M. (1997) Visual pigments and oil droplets from six classes of photoreceptor in the retinas of birds. Vision Res. 37, 2183-2194. doi: 10.1016/s0042-6989(97) 00026-6

Brooke, M., and Davies, N. B. (1988). Egg mimicry by cuckoos Cuculus canorus in relation to discrimination by hosts. Nature 335, 630-632. doi: 10.1038/ 335630a0

Buchanan, K. L., Grindstaff, J. L., and Pravosudov, V. V. (2008). Conditiondependence, developmental plasticity, and cognition: implications for ecology and evolution. Trends Ecol. Evol. 23, 1-7. doi: 10.1016/j.tree.2013.02.004

Canniff, L., Dainson, M., López, A. V., Hauber, M. E., Grim, T., Samaš, P., et al. (2018). Probing the limits of egg recognition using egg rejection experiments along phenotypic gradients. J. Visual. Exp. 138:e57512. doi: 10.3791/57512

Cassey, P., Honza, M., Grim, T., and Hauber, M. E. (2008). The modelling of avian visual perception predicts behavioural rejection responses to foreign egg colours. Biol. Lett. 4, 515-517. doi: 10.1098/rsbl.2008.0279

Caves, E. M., Green, P. A., Zipple, M. N., Peters, S., Johnsen, S., and Nowicki, S. (2018). Categorical perception of colour signals in a songbird. Nature 560, 365-367. doi: 10.1038/s41586-018-0377-7

Caves, E. M., Schweikert, L. E., Green, P. A., Zipple, M. N., Taboada, C., Peters, S., et al. (2020). Variation in carotenoid-containing retinal oil droplets correlates with variation in perception of carotenoid coloration. Behav. Ecol. Sociobiol. 74. doi: 10.1007/s00265-020-02874-5

Cheke, R. A., Venn, J. F., and Jones, P. J. (2006). A spatio-temporal model for predicting when and where red-billed quelea will breed in southern Africa. J. Ornithol. 147, 1-297.

Davies, N. B., and Brooke, L. (1989). An experimental study of co-evolution between the cuckoo, Cuculus canorus, and its hosts. II. host egg markings, 
chick discrimination and general discussion. J. Animal Ecol. 58, 225-236. doi: $10.2307 / 4996$

Dawkins, R., and Krebs, J. R. (1979). Arms races between and within species. Proc. R. Soc. London B 205, 489-511.

Dominoni, D. M., Halfwerk, W., Baird, E., Buxton, R. T., Fernández-Juricic, E., Fristrup, K. M., et al. (2020). Why conservation biology can benefit from sensory ecology. Nat. Ecol. Evol. 4, 502-511. doi: 10.1038/s41559-020-1135-4

Dukas, R., and Waser, N. M. (1994). Categorization of food types enhances foraging performance of bumblebees. Animal Behav. 48, 1001-1006. doi: 10.1006/anbe. 1994.1332

Endler, J. A. (1993). The color of light in forests and its implications. Ecol. Monogr. 63, 1-27. doi: 10.2307/2937121

Endler, J. A., and Mielke, P. W. (2005). Comparing entire colour patterns as birds see them. Biol. J. Linn. Soc. 86, 405-431. doi: 10.1111/j.1095-8312.2005. 00540.x

Endler, J. A., Westcott, D. A., Madden, J. R., and Robson, T. (2005). Animal visual systems and the evolution of color patterns: sensory processing illuminates signal evolution. Evolution 59, 1795-1818. doi: 10.1111/j.0014-3820.2005. tb01827.x

Goldsmith, T. H. (1990). Optimization, constraint, and history in the evolution of eyes. Quart. Rev. Biol. 65, 281-322. doi: 10.1086/416840

Goldstone, R. L., and Hendrickson, A. T. (2009). Categorical perception. Wiley Interdiscipl. Rev. Cogn. Sci. 1, 69-78.

Green, P. A., Brandley, N. C., and Nowicki, S. (2020). Categorical perception in animal communication and decision-making. Behav. Ecol. 31, 859-867. doi: 10.1093/beheco/araa004

Ham, A. D., and Osorio, D. C. (2007). Colour preferences and colour vision in poultry chicks. Proc. R. Soc. B 274, 1941-1948. doi: 10.1098/rspb.2007.0538

Hanley, D., Cassey, P., and Doucet, S. M. (2013). Parents, predators, parasites, and the evolution of eggshell colour in open nesting birds. Evol. Ecol. 27, 593-617. doi: 10.1007/s10682-012-9619-6

Hanley, D., López, A. V., Fiorini, V. D., Reboreda, J. C., Grim, T., and Hauber, M. E. (2019b). Variation in multicomponent recognition cues alters egg rejection decisions: a test of the optimal acceptance threshold hypothesis. Philos. Trans. R. Soc. London B 374:20180195. doi: 10.1098/rstb.2018. 0195

Hanley, D., Gern, K., Hauber, M. E., and Grim, T. (2019a). Host responses to foreign eggs across the avian visual color space. Am. Natur. 194, 17-27. doi: $10.1086 / 703534$

Hanley, D., Grim, T., Cassey, P., and Hauber, M. E. (2015). Not so colourful after all: eggshell pigments constrain avian eggshell colour space. Biol. Lett. 11:20150087. doi: 10.1098/rsbl.2015.0087

Hanley, D., Grim, T., Igic, B., Samaš, P., López, A. V., Shawkey, M. D., et al. (2017). Egg discrimination along a gradient of natural variation in eggshell coloration. Proc. R. Soc. B 284:20162592. doi: 10.1098/rspb.2016.2592

Harnad, S. (1987). "Psychophysical and cognitive aspects of categorical perception: a critical overview," in Categorical Perception: The Groundwork of Cognition (, ed. S. Harnad (New York: Cambridge University Press), 1-25. doi: 10.1016/j. cognition.2009.06.008

Hart, N. S. (2001). The visual ecology of avian photoreceptors. Progr. Retinal Eye Res. 20, 675-703. doi: 10.1016/s1350-9462(01)00009-x

Hauber, M. E., Tong, L., Bán, M., Croston, R., Grim, T., Waterhouse, G. I. N., et al. (2015b). The value of artificial stimuli in behavioral research: making the case for egg rejection studies in avian brood parasitism. Ethology 121, 521-528. doi: 10.1111/eth.12359

Hauber, M. E., Aidala, Z., Igic, B., Shawkey, M. D., and Moskát, C. (2015a). Experimental shifts in egg-nest contrasts do not alter egg rejection responses in an avian host-brood parasite system. Animal Cogn. 18, 1133-1141. doi: 10.1007/s10071-015-0886-9

Hauber, M. E., Kim, C. R., Goethe, C., and Hanley, D. (2020). Self-referent phenotype matching is a poor predictor of egg rejection by American Robins. J. Field Ornithol. 91, 254-262. doi: 10.1111/jofo.12339

Honza, M., Polaèiková, L., and Procházka, P. (2007). Ultraviolet and green parts of the colour spectrum affect egg rejection in the song thrush (Turdus philomelos). Biol. J. Linn. Soc. 92, 269-276. doi: 10.1111/j.1095-8312.2007. 00848.x

Jacobs, G. H. (1981). Comparative Color Vision. Series in Cognition and Perception. New York: Academic Press.
Kelber, A. (2016). Colour in the eye of the beholder: receptor sensitivities and neural circuits underlying colour opponency and colour perception. Curr. Opin. Neurobiol. 41, 106-112. doi: 10.1016/j.conb.2016.09.007

Kelber, A., and Osorio, D. (2010). From spectral information to animal colour vision: experiments and concepts. Proc. R. Soc. B 277, 1617-1625. doi: 10.1098/ rspb.2009.2118

Kepecs, A., Uchida, N., Zariwala, H. A., and Mainen, Z. F. (2008). Neural correlates, computation and behavioural impact of decision confidence. Nature 455, 227231. doi: $10.1038 /$ nature 07200

Krauskopf, J., and Gegenfurtner, K. (1992). Color discrimination and adaptation. Vision Research 32, 2165-2175. doi: 10.1016/0042-6989(92)90077-v

Lachlan, R. F., and Nowicki, S. (2015). Context-dependent categorical perception in a songbird. Proc. Natl. Acad. Sci. U.S.A. 112, 1892-1897. doi: 10.1073/pnas. 1410844112

Langmore, N. E., Kilner, R. M., Butchart, S. H. M., Maurer, G., Davies, N. B., Cockburn, A., et al. (2005). The evolution of egg rejection by cuckoo hosts in Australia and Europe. Behav. Ecol. 16, 686-692. doi: 10.1186/1742-9994-11-34

Lind, O. (2016). Colour vision and background adaptation in a passerine bird, the zebra finch (Taeniopygia guttata). R. Soc. Open Sci. 3:160383. doi: 10.1098/rsos. 160383

Manna, T. J., Hanley, D., Honza, M., Capek, M., Rutila, J., Samaš, P., et al. (2020). Fitting different visual models to behavioral patterns of parasitic egg rejection along a natural egg color gradient in a cavity-nesting host species. Vision Res. 167, 54-59. doi: 10.1016/j.visres.2019.12.007

Nelson, D. A., and Marler, P. (1989). Categorical perception of a natural stimulus continuum: birdsong. Science 244, 976-978. doi: 10.1126/science.2727689

Ödeen, A., and Håstad, O. (2003). Complex distribution of avian color vision systems revealed by sequencing the SWS1 opsin from total DNA. Mol. Biol. Evol. 20, 855-861. doi: 10.1093/molbev/msg108

Olsson, P., Johnsson, R. D., Foster, J. J., Kirwan, J. D., Lind, O., and Kelber, A. (2020). Chicken colour discrimination depends on background colour. J. Exp. Biol. 223:jeb209429.

Olsson, P., Lind, O., and Kelber, A. (2018). Chromatic and achromatic vision: parameter choice and limitations for reliable model predictions. Behav. Ecol. 29, 273-282. doi: 10.1093/beheco/arx133

Osorio, D., and Vorobyev, M. (2018). Principles and application of the receptor noise model of color discrimination: a comment on Olsson et al. Behav. Ecol. $29,283-284$.

Osorio, D., Vorobyev, M., and Jones, C. D. (1999). Colour vision of domestic chicks. J. Exp. Biol. 202, 2951-2959. doi: 10.1242/jeb.202.21.2951

Peer, B. D., and Sealy, S. G. (2004). Correlates of egg rejection in hosts of the Brown-headed Cowbird. Condor 106, 580-599. doi: 10.1007/s00114-0171446-8

Poralla, J., and Neumeyer, C. (2006). Generalization and categorization of spectral colors in goldfish. II. experiments with two and six training wavelengths. J. Comparat. Physiol. A 192, 469-479. doi: 10.1007/s00359-005-0082-5

Price, T., and Fialko, K. (2018). Receptor noise models: time to consider alternatives?: A comment on Olsson et al. Behav. Ecol. 29, 284-285. doi: 10. 1093/beheco/arx155

Price, T. D., Stoddard, M. C., Shevell, S. K., and Bloch, N. I. (2019). Understanding how neural responses contribute to the diversity of avian colour vision. Animal Behav. 155, 297-305. doi: 10.1016/j.anbehav.2019.05.009

Reeve, H. K. (1989). The evolution of conspecific acceptance thresholds. Am. Natur. 133:407. doi: 10.1086/284926

Renoult, J. P., Kelber, A., and Schaefer, H. M. (2015). Colour spaces in ecology and evolutionary biology. Biol. Rev.92, 292-315. doi: 10.1111/brv.12230

Repp, B. H. (1984). "Categorical perception: issues, methods, findings," in Speech and Language: Advances in Basic Research and Practice, Vol. 10, ed. N. J. Lass (New York: Academic Press), 244-335.

Rocha, F. A. F., Saito, C. A., Silveira, L. C. L., de Souza, J. M., and Ventura, D. F. (2008). Twelve chromatically opponent ganglion cell types in turtle retina. Visual Neurosci. 25, 307-315. doi: 10.1017/S0952523808080516

Rothstein, S. I. (1975). An experimental and teleonomic investigation of avian brood parasitism. Condor 77, 250-271.

Sandell, J. H., Gross, C. G., and Bornstein, M. H. (1979). Color categories in macaques. J. Comparat. Physiol. Psychol. 93, 626-635. doi: 10.1037/h0077594

Shepard, R. N. (1987). Toward a universal law of generalization for psychological science. Science 237, 1317-1323. doi: 10.1126/science.3629243 
Soler, J. J., Avilés, J. M., Møller, A. P., and Moreno, J. (2012). Attractive blue-green egg coloration and cuckoo-host coevolution. Biol. J. Linn. Soc. 106, 154-168. doi: 10.1111/j.1095-8312.2012.01857.x

Soler, M., and Soler, J. (2000). Brood-parasite interactions between great spotted cuckoos and magpies: a model system for studying coevolutionary relationships. Oecologia 125, 309-320. doi: 10.1007/s0044200 00487

Spottiswoode, C. N., and Stevens, M. (2010). Visual modeling shows that avian host parents use multiple visual cues in rejecting parasitic eggs. Proc. Natl. Acad. Sci. U.S.A. 107, 8672-8676. doi: 10.1073/pnas.0910486107

Spottiswoode, C. N., and Stevens, M. (2012). Host-parasite arms races and rapid changes in bird egg appearance. Am. Natur. 179, 632-648. doi: 10.1086/665031

Stevens, M. (2011). Avian vision and egg colouration: concepts and measurements. Avian Biol. Res. 4, 168-184.

Stevens, M. (2013). Bird brood parasitism. Curr. Biol. 23, R909-R913.

Stevens, M., Parraga, C. A. I, Cuthill, C., Partridge, J. C., and Troscianko, T. S. (2007). Using digital photography to study animal coloration. Biol. J. Linn. Soc. 90, 211-237. doi: 10.1111/j.1095-8312.2007.00725.x

Stevens, M., Troscianko, J., and Spottiswoode, C. N. (2013). Repeated targeting of the same hosts by a brood parasite compromises host egg rejection. Nat. Commun. 4:2475. doi: 10.1038/ncomms3475

Stoddard, M. C., and Hauber, M. E. (2017). Colour, vision and coevolution in avian brood parasitism. Philos. Trans. R. Soc. B 372:20160339. doi: 10.1098/rstb.2016. 0339

Stoddard, M. C., and Stevens, M. (2011). Avian vision and the evolution of egg color mimicry in the common cuckoo. Evolution 65, 2004-2013. doi: 10.1111/ j.1558-5646.2011.01262.x

Stokke, B. G., Hafstad, I., Rudolfsen, G., Bargain, B., Beier, J., Bigas Campàs, D., et al. (2007). Host density predicts presence of cuckoo parasitism in reed warblers. Oikos 116, 913-922. doi: 10.1111/j.2007.0030-1299. 15832.x

Tapper, D. N., and Halpern, B. P. (1968). Taste stimuli: a behavioral categorization. Science 161, 708-709. doi: 10.1126/science.161.3842.708

ten Cate, C., and Rowe, C. (2007). Biases in signal evolution: learning makes a difference. Trends Ecol. Evol. 22, 380-387.

Treisman, M., Faulkner, A., Naish, P. L., and Rosner, B. S. (1995). Voiceonset time and tone-onset time: the role of criterion-setting mechanisms in categorical perception. Quart. J. Exp. Psychol. A 48, 334-366. doi: 10.1080/ 14640749508401394
Vasas, V., Brebnera, J. S., and Chittka, L. (2018). Color discrimination is not just limited by photoreceptor noise: a comment on Olsson et al. Behav. Ecol. 29, 284-285.

Ventura, D. F., Zana, Y., De Souza, J. M., and Devoe, R. D. (2001). Ultraviolet colour opponency in the turtle retina. J. Exp. Biol. 204, 2527-2534. doi: 10. 1242/jeb.204.14.2527

Vorobyev, M. (2003). Coloured oil droplets enhance colour discrimination. Proc. R. Soc. B 270, 1255-1261. doi: 10.1098/rspb.2003.2381

Vorobyev, M., and Osorio, D. (1998). Receptor noise as a determinant of colour thresholds. Proc. R. Soc. London B 265, 351-358. doi: 10.1098/rspb.1998. 0302

Yang, C., Li, Z., Zhang, Y., Wang, H., Liang, W., and Møller, A. P. (2016). Egg polymorphism and egg discrimination in the Daurian Redstarts Phoenicurus auroreus, a host of the common cuckoo Cuculus canorus. Ornithol. Sci. 15, $127-132$.

Zhang, J., Møller, A. P., Yan, D., Li, J., and Deng, W. (2021a). Egg rejection changes with seasonal variation in risk of cuckoo parasitism in Daurian Redstarts, Phoenicurus auroreus. Animal Behav. 175, 193-200. doi: 10.1016/j.anbehav. 2021.03.007

Zhang, J., Santema, P., Li, J., Yang, L., Deng, W., and Kempenaers, B. (2021b). Host personality predicts cuckoo egg rejection in Daurian Redstarts Phoenicurus auroreus. Proc. R. Soc. B Biol. Sci. 288:20210228. doi: 10.1098/rspb.2021.0228

Conflict of Interest: The authors declare that the research was conducted in the absence of any commercial or financial relationships that could be construed as a potential conflict of interest.

Publisher's Note: All claims expressed in this article are solely those of the authors and do not necessarily represent those of their affiliated organizations, or those of the publisher, the editors and the reviewers. Any product that may be evaluated in this article, or claim that may be made by its manufacturer, is not guaranteed or endorsed by the publisher.

Copyright (c) 2021 Hanley, Rutledge and Villa. This is an open-access article distributed under the terms of the Creative Commons Attribution License (CC BY). The use, distribution or reproduction in other forums is permitted, provided the original author(s) and the copyright owner(s) are credited and that the original publication in this journal is cited, in accordance with accepted academic practice. No use, distribution or reproduction is permitted which does not comply with these terms. 